\title{
Effect of the total fraction of Bacillus subtilis GM5 lipopeptides on the growth parameters and formation of the bacterial microbiota of broiler chickens
}

\author{
Guzel Lutfullina ${ }^{1,{ }^{*}}$, Daria Pudova $^{1}$, Natalia Gogoleva ${ }^{2,3}$, Elena Shagimardanova ${ }^{2}$ and Ayslu \\ Mardanova $^{1}$ \\ ${ }^{1}$ SRL "Microbial biotechnologies", Institute of Fundamental Medicine and Biology, Kazan Federal \\ University, Kazan, Russia \\ ${ }^{2}$ Laboratory "Extreme biology", Institute of Fundamental Medicine and Biology, Kazan Federal \\ University, Kazan, Russia \\ ${ }^{3}$ Kazan Institute of Biochemistry and Biophysics of the Kazan Scientific Center of the Russian \\ Academy of Sciences, Kazan, Russia
}

\begin{abstract}
Intestinal microbes play a key role in the energy metabolism of broiler chickens, participate in the development of the gastrointestinal tract, including the regulation of intestinal epithelial proliferation, vitamin synthesis and ion absorption, fermentation of carbohydrates and proteins, biotransformation of bile acids, protection from pathogens and modulation of the immune system. Metagenomic analysis of the gastrointestinal microbiota allows to find approaches to improve the growth and productivity of chickens by introducing a diet based on beneficial bacterial strains or their secondary metabolites. In this paper, we studied the effect of the total fraction of Bacillus subtilis GM5 lipopeptides on the growth parameters and formation of bacterial communities in the caecum of cross Cobb 500 broiler chickens. It was found that the addition of bacillary lipopeptides to the feed resulted in an increase in chicken weight by $12.7 \%$ and a decrease in feed conversion by 6.36\% compared to the control $(P<$ $0.05)$. It was also shown that the introduction of a feed additive in the form of a lipopeptide fraction modulates the structure of the bacterial microbiota of the caecum of chickens. Thus, the proportion of classes Bacteroidia, Negativicutes, Betaproteobacteria, Epsilonproteobacteria, Deltaproteobacteria, Synergistia in the caecal microbiota of chickens of the experimental group increases, and the proportion of Clostridia, Methanobacteria decreases in comparison with the control.
\end{abstract}

\section{Introduction}

Poultry is one of the most important sources of protein for humans. Presently, the growing demand for food makes it necessary to rapidly increase the production of poultry

\footnotetext{
*Corresponding author: g.h95@mail.ru
} 
meat [1]. Animal health is closely related to the status of the gastrointestinal tract, violation, or dysbiosis of which leads to harmful consequences [2]. The homeostasis and functionality of poultry intestines are influenced by various factors, such as diet, feed additives, housing conditions, and resident intestinal microbiota [3], which includes trillions of microorganisms localized mainly in the distal gastrointestinal tract [4]. These microbial communities mediate feed digestion, control intestinal homeostasis, and prevent the spread of infection. Animal metabolism is a complex process regulated by the genes of both the host and the bacteria that make up the intestinal flora. Thus, intestinal microbiota plays an important role in the health, growth, and metabolism of animals, as well as in the absorption of nutrients. Intestinal flora can be regulated by probiotics, which promote a healthy microbial community by suppressing the growth of pathogenic species, as well as improve feed conversion rates, and reduce the spread of drug-resistant microorganisms, making them a suitable alternative to antibiotics [5].

The bacteria used as probiotics belong to the genera Lactobacillus, Enterococcus, Bacillus, etc. There is a certain problem associated with the sensitivity of most probiotic strains to the conditions of the intestinal environment, which significantly reduces the effect of the supplement [5]. Unlike most other species, spore-forming Bacillus spp. are more resistant to adverse environmental conditions, which makes them promising as feed probiotic additives. Many Bacillus strains are able to restore and maintain the balance of the intestinal flora of chickens, improve immune function, increase resistance to diseases, and improve feed conversion, which ultimately stimulates the growth and development of poultry [6].

Bacillus spp. are producers of 66 different antimicrobial peptides (AMP), some of which have been isolated, purified, and commercialized [5]. The high antagonistic activity of Bacillus against various pathogens is associated with the ability to synthesize a wide range of non-ribosomal AMP, such as the surfactin, iturin, fengicin, polymyxin, and kurstakin lipopeptides, whose activity is mediated by the destruction of bacterial membranes, which makes it difficult to develop resistance [5]. It has been shown that lipopeptides of the fengicin, surfactin and iturin families show antimicrobial activity against pathogenic, opportunistic, and phytopathogenic bacteria and fungi, and are also promising as biological surfactants [6]. However, the effect of antimicrobial lipopeptides on animal models has not been sufficiently studied. Animal studies are important for evaluating the safety of these compounds and determining the potential for future biotechnological production of antimicrobials based on them.

\section{Materials and methods}

\subsection{Preparation of the total lipopeptide fraction}

The total lipopeptide fraction was isolated by acid deposition from the cell-free culture medium of the Bacillus subtilis GM5 strain isolated from the potato rhizosphere. Antimicrobial activity of the total fraction of GM5 lipopeptides in vitro was previously characterized by us [7]. The bacteria were grown on the following composition (g/l) of soy medium nutrition $(\mathrm{SMN})$ : mannitol - 26.2, soy flour $-21.9, \mathrm{NaNO}_{3}-3.1, \mathrm{MnSO}_{4} * 4 \mathrm{H}_{2} \mathrm{O}-$ 0.2 for 72 hours at a temperature of $37^{\circ} \mathrm{C}$ on an IKA ${ }^{\circledR K S} 4000$ shaker thermostat at a swing intensity of $200 \mathrm{rpm}$. The cells were deposited by centrifugation at $14,000 \mathrm{rpm}$ for 30 minutes at $4^{\circ} \mathrm{C}$. The total lipopeptide fraction was isolated from $1000 \mathrm{ml}$ of Bacillus culture fluid. After precipitation, the total lipopeptide fraction was dried on a rotary evaporator and stored at room temperature. 


\subsection{In vivo experiment on broiler chickens}

Model experiments on broiler chickens were carried out in the conditions of the farm "Lachyn" ("KFH Alimucheva Z.I.", Medvedevsky district, Mari El Republic, Russia). For the experiment, 60 day-old broiler chickens of the Cobb 500 cross were obtained from a local commercial incubator (Medvedevsky district, Mari El, Russia). Of these, 2 groups of 30 chickens were formed without gender division. The first group received complete feed, and the second group received the total fraction of Bacillus lipopeptides in addition to the feed. Dried lipopeptide powder was added to the feed by mechanical stirring in an amount of 10-50 mg per $1 \mathrm{~kg}$ of feed. Broiler chickens received lipopeptides from day 1 to day 35 . From day 1 to day 10, broiler chickens received Starter compound feed, from day 11 to day 20 - "Grower", from day 21 to day 35 - "Finisher". Broiler chickens were kept in ventilated cages (100 cm long, $80 \mathrm{~cm}$ wide, $40 \mathrm{~cm}$ high) at controlled room temperature and lighting. All experiments with chickens were conducted in compliance with bioethical standards. Maintenance, feeding, and care of animals and killing of animals for sampling is conducted according to the requirements for the care and use of experimental animals of the Kazan Federal University and the Directive of the European Parliament and of the Council on the protection of animals used for scientific purposes dated September 22, 2010 (Directive 2010/63 / UE about the protection of animals used for scientific purposes).

\subsection{Determining growth parameters of broiler chickens}

During the experiment, the daily weighing of broiler chickens of both groups was performed to determine parameters such as the total weight and average daily weight gain. The amount of feed consumed was determined by measuring the remaining feed on a weekly basis from the beginning of the experiment. The feed conversion rate was calculated by dividing the feed consumed by the bodyweight gain.

\subsection{Metagenomic analysis of caecum contents}

\subsubsection{Sample preparation, DNA isolation, and 16s rRNA gene sequencing}

3 chickens were randomly selected from the control and experimental groups for killing. $200 \mathrm{mg}$ of caecum contents were selected from each chicken in three repeats for genomic DNA isolation using the commercial QIAamp Fast DNA Stool Mini kit (QIAGEN, Germany) according to the manufacturer's instructions. 18 DNA samples were prepared for sequencing: 9 samples each for the control and experimental groups. The concentration and quality of DNA were evaluated using NanoDrop 2000 (Thermo, USA) and gel electrophoresis. The isolated DNA was stored at $-20^{\circ} \mathrm{C}$ until further processing. PCR was performed using Q5 Hot Start High-Fidelity 2X Master Mix (NEB, UK) and universal primers 341F (5-CCTACGGGNGGCWGCAG-3) and 805R (5GACTACHVGGGTATCTAATCC-3) containing multiplex identifiers [8]. The distribution of fragments in the library was evaluated using an Agilent 2100 bioanalyzer (Agilent Technologies, USA), and the concentration was measured using a Qubit 3.0 fluorometer (Thermo Fisher Scientific, USA). Libraries containing 16S rRNA genes were sequenced on the MiSeq platform using the MiSeq v3 panel (Illumina, USA). Sequencing data were analyzed using the QIIME program version 1.5.0. [9]. Quality control and filtering of sequences, combining sequences in OTU (operational taxonomic units) were performed using a homology of $97 \%$. Based on the results obtained, the taxonomic position of OTU was determined using the RPD database (http://rdp.cme.msu.edu). 


\subsubsection{Statistical data processing}

Statistical analysis was performed using two-way ANOVA analysis and Student's tcriterion.

\section{Results and discussion}

\subsection{Study of the effect of the total fraction of $B$. subtilis GM5 lipopeptides on the growth parameters of broiler chickens}

A comparative analysis of the growth parameters of broiler chickens of the control group, which received only basic compound feed from day 1 to day 35, and the experimental group, which received an additional total fraction of lipopeptides as a feed additive, revealed a positive effect of the supplement. On day 35, the average weight gain of control chickens was $1298.0 \pm 49.00 \mathrm{~g}$, and experimental chickens $-1463.0 \pm 76.25 \mathrm{~g}(P<0.05)$, which is $12.7 \%$ higher than the control (Table 1). Feed consumption in chickens of the experimental group was also higher by $5.30 \%$ and amounted to $3227.05 \pm 124.81 \mathrm{~g}$ compared to the control $(3064.57 \pm 115.49 \mathrm{~g})$. The average daily weight gain of chickens in the control and experimental groups was $37.09 \pm 1.40 \mathrm{~g}$ and $41.80 \pm 1.86 \mathrm{~g}$, respectively. Feed conversion in chickens of the experimental group was lower by $6.36 \%$ and amounted to 2.21 units, while in the control group it was 2.36 units (table 1).

Table 1. Growth rates of Cobb 500 cross broiler chickens

\begin{tabular}{|c|c|c|c|}
\hline \multirow[t]{3}{*}{$\begin{array}{c}\text { Time, } \\
\text { day }\end{array}$} & Control group & $\begin{array}{l}\text { Experimental group that received } \\
\text { the total lipopeptide fraction }\end{array}$ & \multirow[t]{2}{*}{$P$ value } \\
\hline & Mean \pm SEM & Mean \pm SEM & \\
\hline & \multicolumn{2}{|c|}{ Mass, [g] } & \\
\hline \multirow[t]{2}{*}{$0-35$} & $1298.0 \pm 49.00^{*}$ & $1463.0 \pm 76.25^{*}$ & 0.0232 \\
\hline & \multicolumn{2}{|c|}{$\begin{array}{l}\text { Feed consumption, }[\mathrm{g}] \\
\end{array}$} & \\
\hline \multirow[t]{2}{*}{$0-35$} & $3064.57 \pm 115.49 * *$ & $3227.05 \pm 124.81 * *$ & 0.0001 \\
\hline & \multicolumn{3}{|c|}{ Feed conversion, [units] } \\
\hline \multirow[t]{2}{*}{$0-35$} & 2.36 & 2.21 & \\
\hline & \multicolumn{3}{|c|}{ Average daily weight gain, $[\mathrm{g}]$} \\
\hline $0-35$ & $37.09 \pm 1.40^{* *}$ & $41.80 \pm 1.86^{* *}$ & 0.0001 \\
\hline
\end{tabular}

The table shows the average value $\pm \mathrm{t}_{0.05}$. SEM. The values of the experimental and control groups significantly differ $(* \mathrm{P}<0.05, * * \mathrm{P}<0.01)$

\subsection{Analysis of data from a metagenomic study of the caecum}

According to the results of a metagenomic study, representatives of 14 bacterial phyla were identified in the profiles of the caecum microbiota on the 35th day of growth of Cobb 500 broiler chickens in the control and experimental groups. It is shown that the bacteria phylum Firmicutes or Bacteroidetes dominate in the caecum of chickens of different groups. Thus, the proportion of phylum Firmicutes bacteria significantly differed between the groups and reached $49.326 \% \pm 6.949 \%$ in the control group, $33.074 \% \pm 1.592 \%$ in the experimental group $(P<0.05)$. In contrast, the proportion of phylum Bacteroidetes was $23 \%$ higher in the experimental group $(46.420 \% \pm 1.887 \%)$ compared to the control group $(37.644 \% \pm 5.714 \%)$ (figure 1). Even more varied microbiota of chickens of both groups according to the presence of bacteria of Actinobacteria and Proteobacteria phylum. Thus, 
the number of Actinobacteria phylum bacteria $(2.146 \% \pm 1.100 \%)$ was 5 times higher in the experimental group compared to the control group $(0.426 \% \pm 0.211 \%)(P<0.05)$. Also, the proportion of Proteobacteria phylum was significantly higher (3 times) in the experimental group $(6.786 \% \pm 1.349 \%)$ compared to the control group $(2.014 \% \pm 1.706$ $\%)(P<0.05)$. The number of Synergistetes phylum bacteria was also 10 times higher in the experimental group compared to the control group $(P<0.05)$ (figure 1$)$. The proportion of Euryarchaeota bacteria, on the contrary, was 2 times higher in the control group $(4.455 \% \pm$ $2.597 \%$ ) compared to the experimental group $(2.100 \% \pm 0.993 \%)$, but the values did not differ significantly. The data obtained by us on the profiles of the caecum phylum of Cobb 500 broiler chickens are comparable with the results of studies obtained by other authors $[10,11]$. For example, in the work of Tan et al. Firmicutes and Bacteroidetes have been shown to be the dominant bacteria in the caecum, accounting for up to $80 \%$ of the total microbiota [12]. It is known that the mammalian intestine is also dominated by Firmicutes and Bacteroidetes phylum bacteria, which are associated with the metabolism of SCFA (short-chain fatty acids) [13]. Firmicutes have been shown to synthesize butyrate and propionate, and Bacteroidetes synthesize propionate, $\alpha$-amylase, $\alpha$-1,2-mannosidase, and endo-1,4- $\beta$-mannosidase [13], and cleave starch and other polymeric substances.

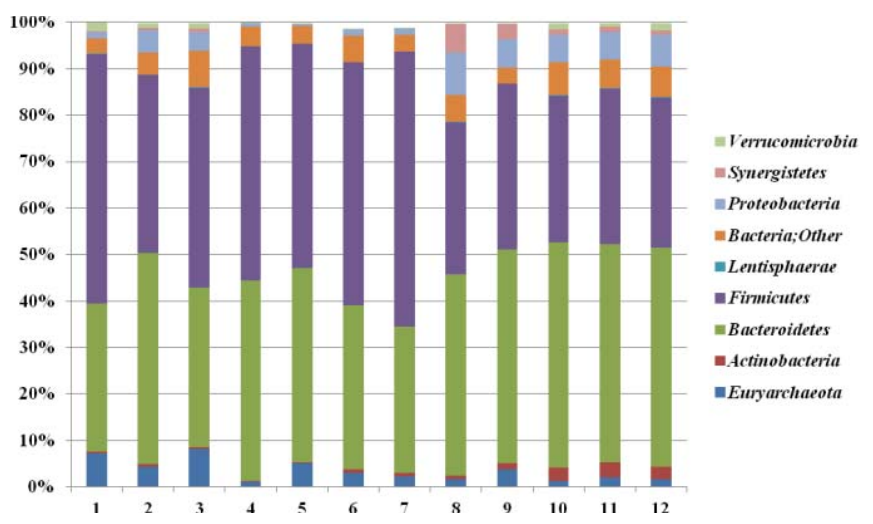

Fig. 1. Representation of the main groups of bacteria at the phylum level in the caecal samples of 35day-old broiler chickens of the control group (1-7) and the experimental group that received a feed additive based on the total lipopeptide fraction (8-12).

On day 35, 27 classes of bacteria were detected in the caecal microbiota of broiler chickens. Despite the high diversity of classes found in the caecum, the dominant 11 classes in the experimental and 5 classes in the control groups accounted for $95 \%$ and $92.5 \%$ of the total microbiota, respectively (figure 2). The class Bacteroidia (ph. Bacteroidetes) was the most numerous in the experimental group, accounting for $48.900 \% \pm 2.324 \%$. Firmicutes phylum was represented by Clostridia $(44.725 \% \pm 4.047 \%$ in the control group and $19.012 \% \pm 1.709 \%$ in the experimental group $(P<0.05))$, Negativicutes $(5.713 \% \pm$ $3.626 \%$ and $10.206 \% \pm 1.787 \%(P<0.05))$ classes, also not classified bacteria $(3.158 \%$ $\pm 0.992 \%$ and $1.987 \% \pm 0.698 \%(P<0.05))$. In the samples of the experimental group that received the total fraction of lipopeptides, Proteobacteria is represented by the classes Betaproteobacteria, Epsilonproteobacteria, and Deltaproteobacteria, the proportion of which is $1.216 \% \pm 0.377 \%, 1.825 \% \pm 0.662 \%, 2.183 \% \pm 0.977 \%$. Synergistetes phylum is represented by a single Synergistia class, the proportion of which is $2.072 \% \pm 0.999 \%$ in the experimental group, and $0.242 \% \pm 0.332 \%$ in the control group $(P<0.05)$ (figure 2$)$. 


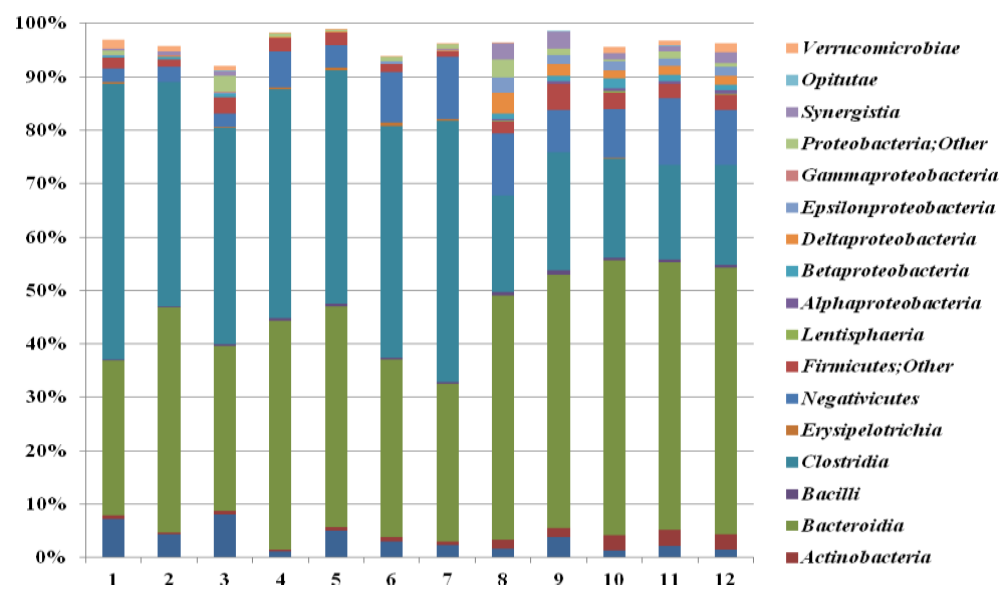

Fig. 2. Representation of the main groups of bacteria at the classes level in the caecum samples of 35day-old broiler chickens of the control group (1-7) and the experimental group that received a feed additive based on the total lipopeptide fraction (8-12). The diagram shows bacteria with a fraction of at least $0.1 \%$.

Representatives of 89 bacterial genera were identified in the microbiota of the control and experimental groups. The intestines of the control group were dominated by the genera Prevotella $(6.208 \% \pm 1.645 \%)$, Methanobrevibacter $(4.411 \% \pm 2.577 \%)$, and the intestines of chickens taking lipopeptides were highly populated by representatives of the genera Bacteroides (39.569\% $1.788 \%)$, Barnesiella (1.406 \% $\pm 0.941 \%)$, Alistipes $(3.225$ $\% \pm 0.645 \%)$. The number of Faecalibacterium bacteria was 4 times higher in the control group compared to the experimental group. But in the experimental group, the number of bacteria of the genera Bifidobacterium and Cloacibacillus was 10 times higher than in the control group $(P<0.05)$. The proportion of bacteria of the Ruminococcus genus (class Clostridia) was at the same level $4.036 \% \pm 0.685 \%$ in the experimental group and $4.157 \%$ $\pm 0.671 \%$ in the control group $(P<0.05)$.

Thus, it was found that the feed additive based on the total fraction of GM5 lipopeptides does not have a negative effect on the growth and development of broiler chickens, but, on the contrary, increases the weight of chickens on the 35th day of growth by $12.7 \%$ compared to the control. The effect of lipopeptides on the qualitative composition of the bacterial microbiota of the chicken caecum is also shown.

\section{Acknowledgment}

This work was supported by the Kazan (Volga region) Federal University and RFBR grant 20-3490130 .

\section{References}

1. S. Leeson, Poult Sci., 91 (2012)

2. M. Matulova, K. Varmuzova, F. Sisak, H. Havlickova, V. Babak, K. Stejskal, et al., BMC Vet Res. 44 (2013)

3. D. Stanley, R.J. Hughes, R.J. Moore, Appl Microbiol Biotechnol, 98 (2014)

4. B. B. Oakley, H. S. Lillehoj, M. H Kogut, W. K. Kim, J. J. Maurer, A. Pedroso, et al., FEMS Microbiol Lett., 360 (2014) 
5. N. Larsen, L. Thorsen, E. N. Kpikpi, et al., Appl. Microb. and Biotech, 98 (2014)

6. N.V. Feoktistova, A.M. Mardanova, G.F. Hadieva, M.R. Sharipova, Uchenye Zapiski Kazanskogo Universiteta. Seriya Estestvennye Nauki, 159 (2017)

7. G. F. Hadieva, M. T. Lutfullin, D. S. Pudova, Y. A. Akosah, N. E. Gogoleva, E. I. Shagimardanova, A. M. Mardanova , M. R. Sharipova, Data in Brief 23 (2019)

8. D. P. Herlemann, M. Labrenz, K. Jürgens, S. Bertilsson, J. J. Waniek, A. F. Andersson, ISME J. 5 (2011)

9. J. Caporaso, J. Kuczynski, J. Stombaugh, K. Bittinger, F. Bushman, E. Costello, Nat Meth., 7 (2010)

10. Y. Shang, S. Kumar, B. Oakley, W.K. Kim, Front Vet Sci., 5 (2018)

11. S. Yadav, R. Jha, J Anim Sci Biotechnol, 10 (2019)

12. Z. Tan, L. Luo, X. Wang, Q. Wen, L. Zhou, K. Wu, PLoS ONE, 14 (2019)

13. O. Polansky, Z. Sekelova, M. Faldynova, A. Sebkova, F. Sisak, I. Rychlik, Appl. and environ. microb., 82 (2016) 